DOI $10.35381 / \mathrm{cm} \cdot \mathrm{v} 7 \mathrm{i} 2.610$

\title{
Website para gestión de promociones y ventas en empresas educativas
}

Website for promotions and sales management in educational companies

\author{
Luis Orlando Albarracín-Zambrano \\ uq.luisalbarracin@uniandes.edu.ec \\ Universidad Regional Autónoma de los Andes, Quevedo \\ Ecuador \\ https://orcid.org/0000-0002-3164-5229 \\ Camilo Ramírez-Saldivias \\ sq.camiloars81@uniandes.edu.ec \\ Universidad Regional Autónoma de los Andes, Quevedo \\ Ecuador \\ https://orcid.org/0000-0002-0867-9498 \\ Neiser Gamarra-Rodríguez \\ sq.neiserogr67@uniandes.edu.ec \\ Universidad Regional Autónoma de los Andes, Quevedo \\ Ecuador \\ https://orcid.org/0000-0002-5574-0255 \\ Josué Anzules-Céspedes \\ sq.josuemac16@uniandes.edu.ec \\ Universidad Regional Autónoma de los Andes, Quevedo \\ Ecuador \\ https://orcid.org/0000-0001-9563-9664
}

Recepción: 15 de septiembre 2021

Aprobación: 15 de noviembre 2021 
Luis Orlando Albarracín-Zambrano; Camilo Ramírez-Saldivias; Neiser Gamarra-Rodríguez; Josué Anzules-Céspedes

\section{Estimado Editor (a):}

En la actualidad hay diferentes páginas web en donde se hacen publicidad sobre las escuelas de danza, hay páginas que no tienen una buena estructura e interfaz con una información detallada de la escuela por tal motivo nuestra página web muestra de manera ordenada la información de la escuela para que así el cliente no tenga ningún inconveniente al momento de realizar la búsqueda de información. El mercadeo educativo es pieza clave para identificar las demandas sociales enunciadas y señalar los propósitos y objetivos que debe perseguir la organización educativa en el contexto en el que se desarrolla. (Ospina Díaz y Sanabria Rangel,2010, p.103).

Al respecto, de acuerdo con Manes (2004), citado por (Gordillo et. al.,2020, p.3) las instituciones educativas están inmersas en un proceso de cambio que quizá sea demasiado veloz para sus tiempos institucionales. Sin embargo, es necesario que las personas a cargo asuman la real utilidad de nuevas herramientas que, como el marketing, ayudan a mejorar la gestión institucional y coadyuvan al proceso de cambio.

Desde la nueva perspectiva institucional, las escuelas deben reconocer que:

a) El marketing educativo es complementario de una gestión administrativa eficiente.

b) Hay limitaciones del uso del marketing por parte de los establecimientos educativos, sobre todo en la educación formal.

c) Existe un conocimiento limitado del marketing por parte de las instituciones educativas y su correcta aplicación.

d) Hay una diferencia entre el marketing mínimo, el marketing equilibrado y el marketing comercial.

e) En las instituciones educativas existen diferentes estructuras culturales, historia y propósitos.

f) Es necesario evaluar de las decisiones administrativas y organizacionales dentro del marco de la ética. 
Luis Orlando Albarracín-Zambrano; Camilo Ramírez-Saldivias; Neiser Gamarra-Rodríguez; Josué Anzules-Céspedes

g) Es necesario que los directivos comprendan la necesidad de capacitación en temas de marketing y management (gerenciamiento) educativo.

h) Es necesario entender que hoy la institución educativa debe incorporar el marketing como proceso y designar un responsable del área al servicio de la comunidad. Por otro lado, un sitio web para mejorar la gestión de promociones y ventas de la escuela de danza "Down Town Studio" de la ciudad de Quevedo", puede brindar una mejor información sobre la escuela de danza y realizar ventas en online para de esta manera genera ingresos para la escuela. En este sentido, para Berzal, Cubero, y Cortijo, 2005, citado por Santa María Vílchez (2018, p.22);

Se denominan aplicaciones web a aquellas aplicaciones cuya interfaz se construye a partir de páginas web. Las páginas web no son más que ficheros de texto en un formato estándar denominado HTML. Estos ficheros se almacenan en un servidor web al cual se accede utilizando el protocolo HTTP, uno de los protocolos de Internet.

Desde el momento en que la empresa decide adoptar internet en su estrategia corporativa, inicia procesos de cambio en su política de hacer negocios, modifica sus decisiones en términos de costos y tiempo. (Barrientos Felipa,2017, p.44)

Así mismo, Perdigón Llanes, Viltres Sala y Madrigal Leiva (2018) indican que:

El crecimiento de Internet tiene un gran potencial y reduce los costos de la entrega de productos y servicios y sobrepasa los límites geográficos para facilitar el intercambio entre compradores y vendedores (Gangeshwer, 2013). Este desarrollo ha provocado el surgimiento de nuevos modelos de negocios, entre los que se destacan los negocios electrónicos, el comercio electrónico y el marketing digital. (p.193)

Los compradores potenciales se multiplican exponencialmente, al ser internet una red global. Para el comprador, las ventajas son también enormes; básicamente puedes acceder a una selección enorme de productos sin desplazarte, desde la tranquilidad de tu casa, comparar un producto con otros similares, ver las opiniones de otros compradores anteriores, sus características y todo ello sin la presión de un vendedor, ni 
Luis Orlando Albarracín-Zambrano; Camilo Ramírez-Saldivias; Neiser Gamarra-Rodríguez; Josué Anzules-Céspedes

colas, ni desplazamientos. Por lo tanto Cedeño Velasco, Asencio Cristóbal y Villegas Alava (2019) manifiestan que:

Se identifica que todo negocio debe ser en base a la gestión ya que se articula los recursos humanos, la parte financiera, los equipos recursos y los propios procesos que deberán cumplir como resultado llenar las expectativas del mercado (cliente) el que acorde a sus satisfacciones dará la continuidad del consumo. (p.193)

Por lo tanto, la Website para gestión de promociones y ventas en empresas educativas involucra varias acciones que permiten una mejor difusión y comercialización de los productos y servicios ofrecidos al público. En tal sentido, Santa María Vílchez (2018) destaca:

Es importante mencionar que una página Web puede contener elementos que permiten una comunicación activa entre el usuario y la información. Esto permite que el usuario acceda a los datos de modo interactivo, gracias a que la página responderá a cada una de sus acciones. (p.23)

De allí la necesidad de la utilización y el apoyo web para mejorar la gestión en ventas y servicios ofrecido por las empresas educativas.

\section{FINANCIAMIENTO}

No monetario.

\section{AGRADECIMIENTO}

A la Universidad Regional Autónoma de los Andes, Quevedo, por motivar el desarrollo de la Investigación. 


\section{REFERENCIAS CONSULTADAS}

Barrientos Felipa, P. (2017). Marketing + internet $=$ e-commerce: oportunidades y desafíos. [Marketing + internet $=$ e-commerce: opportunities and challenges]. Revista Finanzas y Política Económica, 9(1), 4156. https://doi.org/10.14718/revfinanzpolitecon.2017.9.1.3

Cedeño Velasco, A., Asencio Cristóbal, L., y Villegas Alava, M. (2019). Las estrategias gerenciales como base fundamental para la administración en los negocios. [Management strategies as a fundamental basis for management in business]. Revista Universidad y Sociedad, 11(5), 191-200. Recuperado de: https://n9.cl/cw4md

Gordillo, L., Domínguez, B., Vega, C., De la Cruz, A., y Ángeles, M. (2020). El marketing educativo como estrategia para la satisfacción de alumnos universitarios. [Educational marketing as a strategy for the satisfaction of university students]. Propósitos $y$ Representaciones, 8(spe), e499. https://dx.doi.org/10.20511/pyr2020.v8nspe1.499

Ospina Díaz, M., y Sanabria Rangel, P. (2010) Un enfoque de mercadeo de servicios educativos para la gestión de las organizaciones de educación superior en Colombia: el modelo MIGME. [An educational services marketing approach for the management of higher education organizations in Colombia: the MIGME model]. Revista de la Facultad de Ciencias Económicas de la Universidad Militar Nueva Granada, 18(2),107-136. Recuperado de: https://n9.cl/tf84d

Perdigón Llanes, R., Viltres Sala, H., y Madrigal Leiva, I. (2018). Estrategias de comercio electrónico y marketing digital para pequeñas y medianas empresas. [E-commerce and digital marketing strategies for small and medium-sized businesses]. Revista Cubana de Ciencias Informáticas, 12(3), 192-208. Recuperado, de: https://n9.cl/81xt9

Santa María Vílchez, C. (2018). Implementación de un sistema web adaptativo para apoyar en la gestión comercial utilizando el método de costo promedio en la empresa Ferrotumi s.a.c. [Implementation of an adaptive web system to support commercial management using the average cost method in the company Ferrotumi s.a.c]. Tesis de Pregrado. Universidad Católica Santo Toribio de Mogrovejo. Chiclayo. Recuperado de: https://n9.cl/8xfmw 
Revista Interdisciplinaria de Humanidades, Educación, Ciencia y Tecnología

Año VII. Vol. VII. N². Edición Especial II. 2021

Hecho el depósito de ley: pp201602FA4721

ISSN-L: 2542-3029; ISSN: 2610-802X

Universidad Nacional Experimental Francisco de Miranda (UNEFM). Santa Ana de Coro. Venezuela

Luis Orlando Albarracín-Zambrano; Camilo Ramírez-Saldivias; Neiser Gamarra-Rodríguez; Josué Anzules-Céspedes

(C2021 por los autores. Este artículo es de acceso abierto y distribuido según los términos y condiciones de la licencia Creative Commons Atribución-NoComercial-Compartirlgual 4.0 Internacional (CC BY-NC-SA 4.0)

(https://creativecommons.org/licenses/by-nc-sa/4.0/). 